\title{
Tracking ultrashort pulses through dispersive media: Experiment and theory
}

\author{
H. Gersen,* J. P. Korterik, N. F. van Hulst, and L. Kuipers ${ }^{\dagger}$ \\ Applied Optics Group, Department of Science \& Technology and MESA ${ }^{+}$Research Institute, University of Twente, P.O. Box 217, \\ 7500 AE Enschede, The Netherlands
}

(Received 19 March 2003; published 15 August 2003)

\begin{abstract}
We report on the direct visualization of a femtosecond pulse propagating through a dispersive waveguide at a telecom wavelength. The position of a propagating pulse is pinpointed at a particular point in space and time using a scanning probe based measurement. The actual propagation of the pulse is visualized by changing the reference time. Our phase-sensitive and time-resolved measurement provides local information on all properties of the light pulse as it propagates, in particular its phase and group velocity. Here, we show that the group velocity dispersion can be retrieved from our measurement by developing an analytical model for the measurements performed with a time-resolved photon scanning tunneling microscope. As a result, interesting and useful effects, such as pulse compression, pulse spreading, and pulse reshaping, become accessible in the local measurement.
\end{abstract}

DOI: 10.1103/PhysRevE.68.026604

PACS number(s): 42.25.-p, 07.60.Ly, 07.79.Fc, 42.65.Tg

\section{INTRODUCTION}

Light as an electromagnetic wave is characterized by a combination of time varying electric and magnetic fields propagating through space. In the past decades the use of time varying optical fields in the form of ultrashort light pulses has proven to be a valuable tool in the study of ultrafast phenomena [1]. The attractiveness of ultrashort light pulses not only lies in the possibility to trace processes in their ultrafast dynamics but also in the fact that one simply can do things faster. Of primary importance are data transfer and data processing utilizing the large attainable bandwidth. In this respect, probably one of the most spectacular goals is the creation of an all-optical computer.

Optical delay elements capable of transmitting ultrashort pulses will be one of the key ingredients for all-optical data processing [2]. Photonic crystals ( $\mathrm{PhCs}$ ) in which photons experience multiple reflections are one of the promising candidates for this task, as the group velocity in PhCs can be several orders of magnitude lower than in bulk materials with the same refractive index [3-5]. PhCs are materials with spatial periodicity in their refractive index and have dispersion properties that can be tailor made by selecting their scale and geometry [6]. In this way it becomes possible to control the group and phase velocity of ultrashort light pulses in a unique way. The unique dispersion characteristics of PhCs may lead to a variety of interesting optical nonlinear phenomena such as gap solitons [7,8], pulse splitting of generated second-harmonic pulses [9], highly efficient wavelength conversion, and can also be employed for dispersion management in optical telecommunications.

To integrate ultrashort pulses with various evolving photonic technologies, it is necessary to analyze the interaction of ultrashort optical pulses with these photonic devices.

\footnotetext{
*Corresponding author. Email address: h.gersen@tnw.utwente.nl

${ }^{\dagger}$ Present address: Nanophotonics Group, FOM-Institute for Atomic and Molecular Physics (AMOLF), Kruislaan 407, 1098 SJ Amsterdam, The Netherlands.
}

Many investigations on dynamical effects in (non)linear dispersive media are based on numerical simulations [10-12]. We intend to gain a better understanding of the interaction of light with (sub)wavelength sized structures by experimental means.

Experimental investigations on the propagation of ultrashort pulses in (non)linear dispersive media have so far largely been limited to "black-box-type" characterization of the medium [5,13-17]. The known incoming pulse and the measured transmitted or reflected pulse are compared with a theoretical model. By cutting slices from the medium ("cutback" method) it becomes possible to study aspects of the internal pulse development [18]; yet this method is destructive and has several other clear drawbacks. First, not every photonic structure can be arbitrarily changed in length without affecting its properties. Second, if a disagreement between experiment and theory is found, it may be hard to find the underlying cause for the discrepancy. Third, it is important to realize that the black-box method integrates all the pulse propagation effects accumulated in the entire structure. If a structure has spatially varying optical properties, only averaged information is obtained. To overcome these drawbacks and obtain full local information on pulse propagation throughout a medium, local time-resolved measurements are crucial [15]. An experimental method enabling the observation of dynamical effects directly inside a photonic structure has only recently been demonstrated [19].

In the last few years a so-called interferometric photon scanning tunneling microscope (PSTM) has proven its unique capacity to measure the amplitude and phase distribution of the optical field locally inside photonic structures [20-22]. Here, we report on the nondestructive visualization of a femtosecond pulse propagating through a linear dispersive waveguide in space and time using an interferometric PSTM at infrared wavelengths. The ability to measure at a wavelength range relevant for telecommunications opens up the possibility to investigate local dynamic behavior of photonic crystals and integrated optical circuits in the near future. An advantage of the short laser pulses applied is that different effects such as reflection at the end facets (Fabry- 


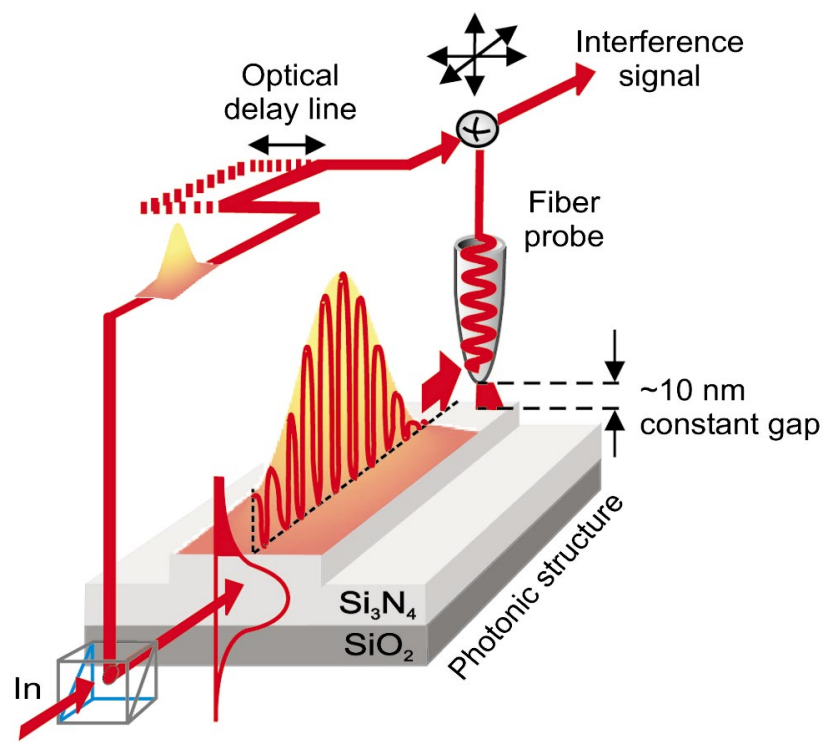

Perot effect) and scattering out of the structure can be discriminated in the time domain.

The microscope used is based on the heterodyne interferometric PSTM, recently developed by our group [23] and provides the full amplitude and phase information of a pulse traveling through a waveguide structure. From these timedependent and phase-sensitive measurements, both the local
FIG. 1. (Color) Schematic representation of a pulse tracking experiment. The evanescent field of the pulse traveling inside the waveguide is picked up by a fiber probe with subwavelength dimensions. The photon tunneling signal picked up by the probe is interferometrically mixed with part of the same pulse that has propagated through the reference branch. The length of the reference branch, and thus the time taken by the reference pulse to travel through this branch, is controlled by an optical delay line. Each subsequent measurement shown in this paper is obtained by raster scanning the optical probe across the photonic structure while the height above the structure $(<10 \mathrm{~nm})$ is kept constant by a feedback mechanism. The simultaneous measurement of optics and topography makes it possible to directly relate optical information to the structural properties.
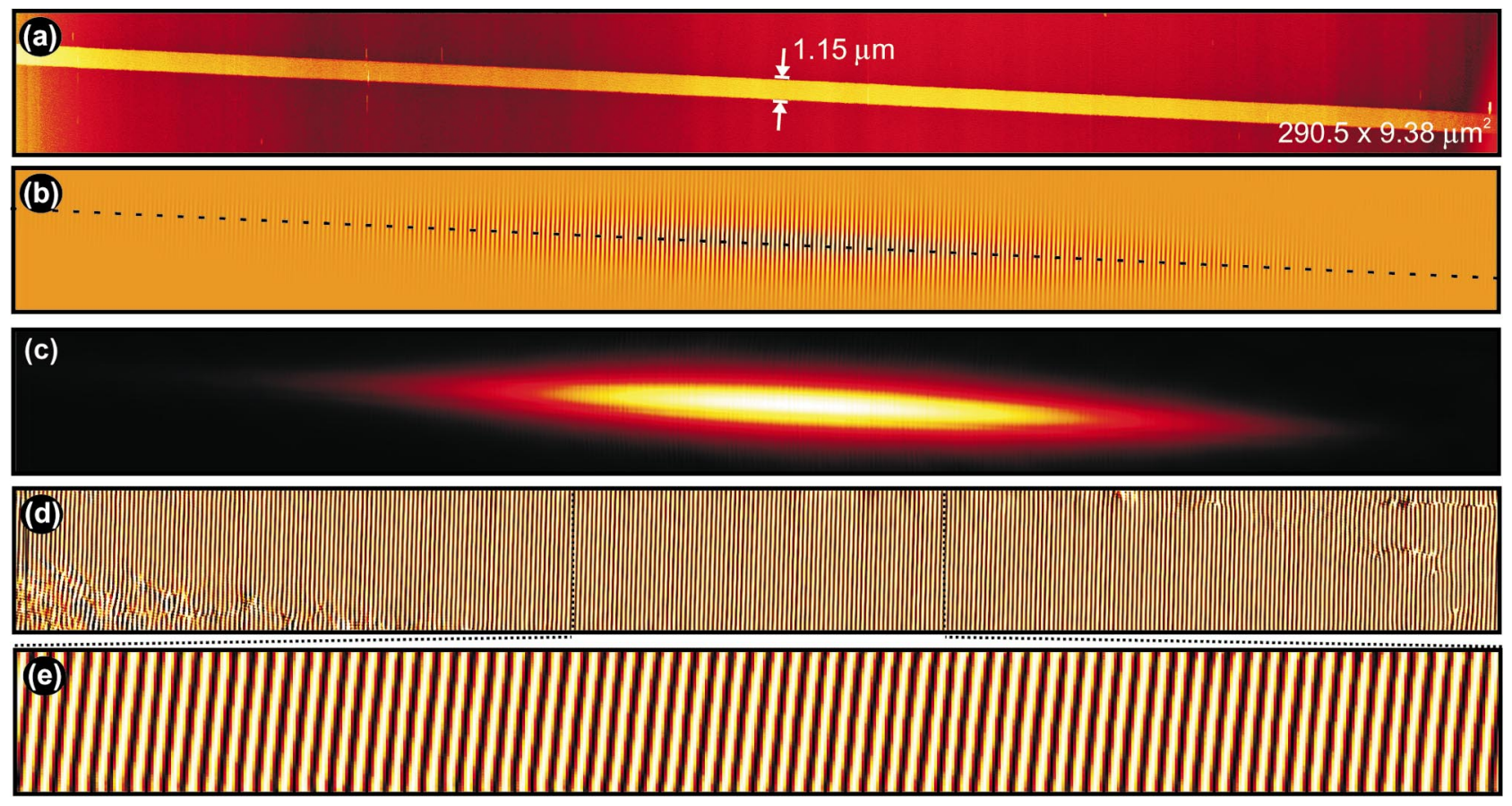

FIG. 2. (Color) A time-resolved heterodyne interference PSTM measurement on a $\mathrm{Si}_{3} \mathrm{~N}_{4}$ channel waveguide for a fixed position of the optical delay (image size of $290.5 \times 9.38 \mu \mathrm{m}^{2}$ ). Linearly polarized light has been coupled in the waveguide to excite the TE 00 mode. (a) The topography of the waveguide. The measured height and width of the waveguide are $39 \pm 3 \mathrm{~nm}$ and $1.15 \pm 0.04 \mu \mathrm{m}$. The slab thickness is determined to be $170 \pm 5 \mathrm{~nm}$. (b) The raw data from the lock-in amplifier, corresponding to the optical amplitude $\mathcal{A}$ times the cosine of the optical phase for a single tracked pulse as a function of the lateral position in the plane of the sample. (c) The optical field amplitude of the pulse while propagating through the waveguide as derived from (b). It is apparent that the amplitude is confined to the waveguide. (d) The corresponding $\cos \Phi$ of the optical field for the complete measurement. (e) A zoom-in on the boxed area given in (d) to clearly show the individual wavefronts (image size: $72.6 \times 9.38 \mu \mathrm{m}^{2}$ ). It is clear that the wave fronts in the image are straight, indicating plane wave propagation corresponding to the $\mathrm{TE}_{00}$ mode. 


\section{EXPERIMENTAL ASPECTS}

A schematic overview of the pulse tracking experiment is depicted in Fig. 1. Linearly polarized light is coupled into an integrated waveguide structure and propagates through the photonic structure. In the PSTM, the local evanescent field above the structure, with a decay length of typically 10-100 $\mathrm{nm}$, is picked up by a fiber probe with subwavelength dimensions. The evanescent field is locally converted into a propagating wave, which is coupled into the fiber probe, guided through the fiber, and subsequently detected. For each position on the photonic structure, the phase and time information of the evanescent field is the same as that of the propagating wave. By picking up the evanescent field, direct information is therefore obtained on the propagating light field. By raster scanning the probe, using a height feedback mechanism, the optical field distribution is probed in the near field of the waveguide surface, while simultaneously the surface topography is acquired.

As an electromagnetic field is characterized by an amplitude, a phase, and a polarization state, it is essential to use coherent detection methods. Therefore the sample and the PSTM have been integrated in one branch of a MachZehnder-type interferometric setup as shown in Fig. 1. At each position on the sample surface, the photon tunneling signal picked up by the near-field probe is interferometrically mixed with light split from the same laser source that has propagated along the reference branch of the setup. The interference between light in the signal and reference branch is measured with a photodetector. The optical frequency of the reference beam is shifted with the difference frequency of 40 $\mathrm{kHz}$ between two acousto-optic (AO) modulators to allow heterodyne detection of the photon tunneling signal. The resulting signal, measured with a dual-output lock-in amplifier (LIA), contains both the optical amplitude and relative phase of the local optical field within the sample [23].

In order to visualize the propagation of a femtosecond pulse, we incorporated an optical delay line in the reference branch of the interferometric setup. Optical interference will only occur when there is temporal overlap between the pulse in the signal and the pulse in the reference branch at the point where the branches join again. In the current experiment the photon-tunneling signal and the reference signal recombine in a 50/50 fiber coupler after propagating through different lengths of a single-mode fiber and other bulk optical glass components. The pulse in the signal branch travels $69.7 \pm 0.5 \mathrm{~cm}$ through dispersive fibers and other glass components (including the sample), and $23.5 \pm 0.2 \mathrm{~cm}$ through air. For the reference branch these distances are 38.5 $\pm 0.5 \mathrm{~cm}$ and $75.6 \pm 0.5 \mathrm{~cm}$, respectively. Note that in the reference branch the path length in air is changed as a function of the position of the optical delay line. The position of the optical delay determines the optical path length of the reference branch, and with this defines a reference time for the measurement.

As a model system for our measurements, we have used a $\mathrm{Si}_{3} \mathrm{~N}_{4}$ planar channel waveguide fabricated in a $\mathrm{Si}_{3} \mathrm{~N}_{4} / \mathrm{SiO}_{2}$ layer system on a $\mathrm{Si}$ substrate. The experimentally determined slab thickness, width, and height of the structure are
$170 \pm 5 \mathrm{~nm}, 1.15 \pm 0.04 \mu \mathrm{m}$, and $39 \pm 3 \mathrm{~nm}$, respectively. Linearly polarized light with a center wavelength of 1300 $\pm 2 \mathrm{~nm}$ has been coupled into the channel waveguide with a polarization parallel to the sample plane, such that only the fundamental $\mathrm{TE}_{00}$ mode is excited. This is, as determined by an effective index method, the only mode supported by the waveguide for the measured waveguide parameters. All measurements have been performed by raster scanning an uncoated fiber probe with the fast axis along the waveguide channel (line frequency $=0.098 \mathrm{~Hz}$ ), for a fixed position of the optical delay line in the reference branch.

The pulses launched into the photonic structure are generated by a Ti:sapphire-pumped optical parametric oscillator (Spectra-Physics Opal). The laser system has a repetition rate of $80 \mathrm{MHz}$. The arrival time $(12.5 \mathrm{~ns})$ between subsequent laser pulses is such that the pulse picked up by the probe can only interfere with part of the same laser pulse that has propagated along the reference branch. The pulse duration of the input field $\widetilde{E}(t)$ is measured by a conventional background free intensity autocorrelation technique and yields a FWHM of the pulse amplitude of $123 \pm 3$ fs [1].

\section{PULSE TRACKING IN A WAVEGUIDE}

A local time-resolved heterodyne interference measurement of a short optical pulse inside our model system is presented in Fig. 2 for a fixed position of the delay line. Figure 2(b) shows the raw data obtained with the LIA, while Fig. 2(a) shows the simultaneously acquired topography. The signal from the LIA corresponds to the measured optical amplitude $\mathcal{A}$ times the cosine of the phase of the optical field $(\cos \Phi)$ of a single tracked pulse, as will be discussed later. Note that a scanning probe based technique is inherently slow so that averaging over many individual laser pulses takes place. From this measurement the optical amplitude and the cosine of the phase of the optical field of the pulse can be separated, as given in Fig. 2(c) and 2(d), respectively. The pattern in Fig. 2(c) shows a roughly Gaussian shape along the propagation direction with a full width at half maximum (FWHM) of $66.8 \mu \mathrm{m}$. The profile of the amplitude in the direction perpendicular to the propagation direction corresponds to the mode profile of an excited $\mathrm{TE}_{00}$ mode. The $\cos \Phi$ term of the optical field as depicted in Fig. 2(d) shows a clear fringe pattern as a function of the lateral position in the plane of the sample. To show the individual wavefronts, a zoom in on the boxed area in Fig. 2(d) is depicted in Fig. 2(e). It is clear that the wavefronts in the image are straight, also indicating single-mode propagation. For multiple modes with different effective indices, the simultaneous detection would lead to a beating pattern in the $\mathcal{A} \cos \Phi$ image [20,23].

The line profile of Fig. 3(a) shows the measured $\mathcal{A} \cos \Phi$ along the dashed line in Fig. 2(b). A zoom in on a part of the line profile is depicted in Fig. 3(b), which shows that individual fringes are clearly resolved. A simple Fourier transform [Fig. 3(c)] of the data along the waveguide suffices to determine which wavelengths are contained in the fringe pattern. We find a central wavelength of $871 \pm 2 \mathrm{~nm}$ inside the structure associated with the central optical frequency $\omega_{0}$ of 


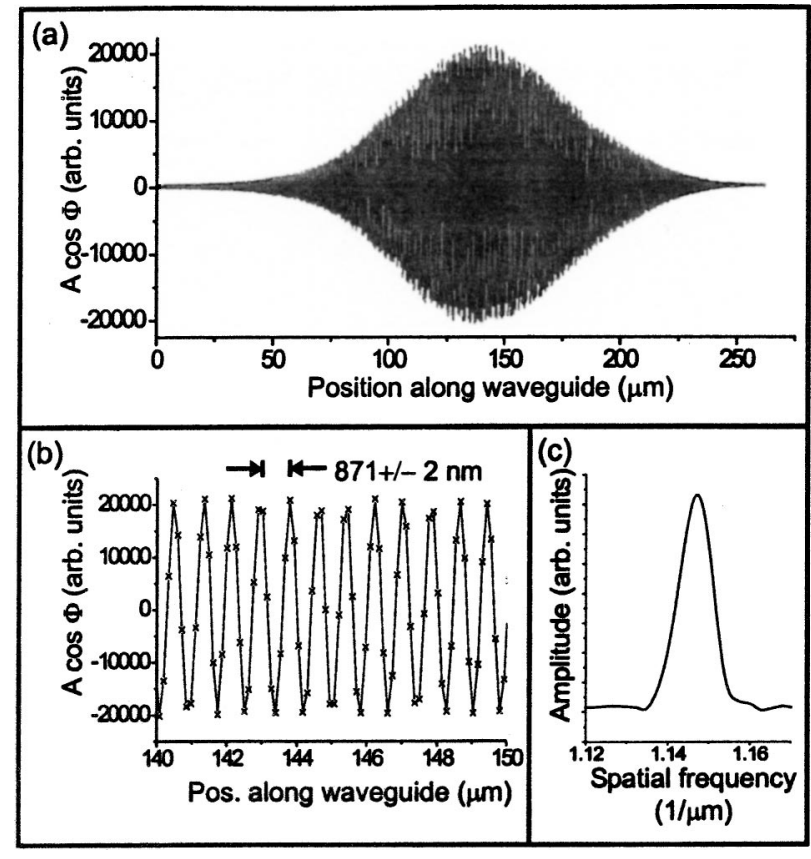

FIG. 3. (a) The optical amplitude times the cosine of the optical phase for a pulse as measured through the heart of the waveguide for the entire scan range [Fig. 2(a)]. (b) A zoom-in on a part of the line profile of (a) from which a period of $871 \pm 2 \mathrm{~nm}$ can be determined by a simple Fourier transform as seen in (c). The periodicity of the wave fronts yields the wavelength inside the structure associated with the optical carrier-frequency cycles. This wavelength corresponds to a phase velocity $v_{\phi}$ of $2.01 \pm 0.02 \times 10^{8} \mathrm{~m} / \mathrm{s}$.

the spectrum of the femtosecond laser pulse. This wavelength corresponds to a phase velocity $v_{\phi}$ of $2.01 \pm 0.02$ $\times 10^{8} \mathrm{~m} / \mathrm{s}\left(n_{\text {eff }}=1.49 \pm 0.01\right)$.

We directly measured the velocity at which the carrierfrequency cycles move forward locally inside the waveguide. However, the envelope of the pulse moves with a different velocity. In Figs. 4(b)-(h) the measured optical amplitudes for seven subsequent measurements with increasing reference times are shown. For each reference time a Gaussian envelope is found, which reveals the position of the pulse, thereby pinpointing the pulse position for each reference time. As time passes, the pulse is found further along the waveguide. The speed at which the pulse envelope propagates can be directly determined from the positional change of the center of the pulse during the known time interval. Between each measurement, the reference time is shifted $200 \pm 2$ fs by lengthening the reference branch by 60.0 $\pm 0.6 \mu \mathrm{m}$. The linear dependence of the position of the pulse in the waveguide as a function of the reference time (Fig. 5) shows that the pulse propagates locally with a constant group velocity. From the slope of the fitted straight line, we find a group velocity $v_{g}$ of $1.67 \pm 0.03 \times 10^{8} \mathrm{~m} / \mathrm{s}$.

The measured group and phase velocity can be compared to the values calculated by an effective index method. These calculations are based on measured refractive indices for the $\mathrm{Si}_{3} \mathrm{~N}_{4}$ and $\mathrm{SiO}_{2}$ layers grown in our institute in the wavelength range $600-1600 \mathrm{~nm}$. The calculations use the locally measured width and height of the ridge of $1.15 \pm 0.04 \mu \mathrm{m}$

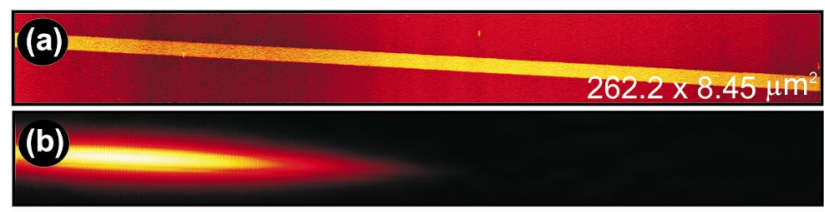

\section{(c)}

(d)

(e)

(f)

\section{(g)}

(h)

FIG. 4. (Color) Pulse tracking experiment for different reference times. Linearly polarized light has been coupled in the waveguide to excite the $\mathrm{TE}_{00}$ mode. (a) The simultaneously measured topography (image size: $262.2 \times 8.45 \mu \mathrm{m}^{2}$ ). (b) $-(\mathrm{h})$ The optical field amplitude as measured by the instrument for different positions of the optical delay line. From (b) to (h) the optical path length of the reference branch is increased in steps of $60 \pm 0.6 \mu \mathrm{m}$. This results in steps of the reference time of $200 \pm 2 \mathrm{fs}$. The measurement shows that the position of the pulse at a reference time can be pinpointed in space. The pulses can be seen to propagate through the structure as a function of time giving a direct local measurement of the group velocity.

and $39 \pm 3 \mathrm{~nm}$, respectively. The calculated effective refractive indices $n_{\text {eff }}$ for the $\mathrm{TE}_{00}$ mode at the wavelength range $600-1600 \mathrm{~nm}$ has been fitted by a quadratic function. The derivative of this function at $1300 \mathrm{~nm}$ then gives a reasonable approximation to the group velocity $v_{g}$ $=1.56 \times 10^{8} \mathrm{~m} / \mathrm{s}$, while the refractive index itself yields a phase velocity $v_{\phi}=1.95 \times 10^{8} \mathrm{~m} / \mathrm{s}$, both in reasonable agreement with the measured values.

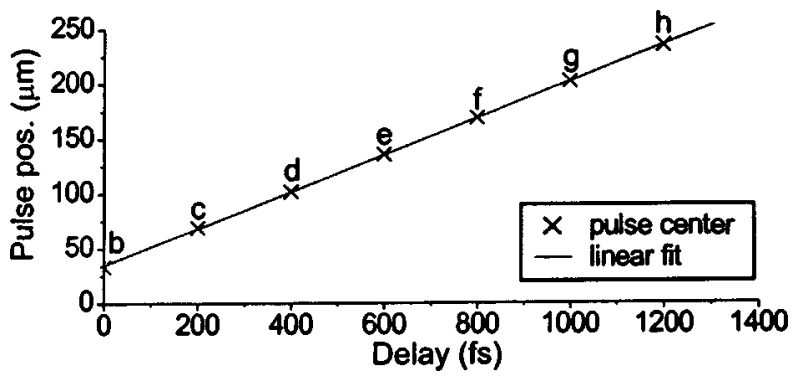

FIG. 5. Measured position of the pulse as a function of the position of the optical delay line (crosses). The letters correspond to the subsequent measurements as given in Fig. 4. The solid straight line represents a least-squares fit to the measured points. The slope of the line shows a local value of the group velocity $v_{g}$ for the pulse of $1.67 \pm 0.03 \times 10^{8} \mathrm{~m} / \mathrm{s}$. 


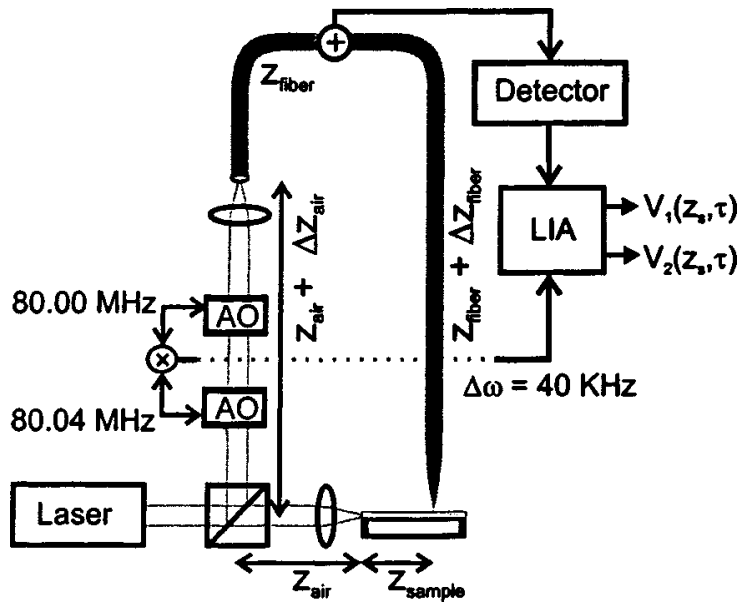

FIG. 6. Mach-Zehnder model for the time-resolved photon scanning tunneling microscope. The fiber length difference $\Delta z_{\text {fiber }}$ between the two arms is compensated by the air path length difference $\Delta z_{\text {air }}$. Heterodyne detection is applied, using acousto-optic modulation of the light in the reference branch. The resulting interference signal is measured with a LIA and allows one to extract the amplitude and phase of the local optical field: AO stands for acoustooptic modulator; and LIA for lock-in amplifier.

\section{THE EFFECT OF DISPERSION}

In the preceding section we have demonstrated how we can locally measure the group and phase velocity of a propagating ultrashort pulse. However, we must take into account that the temporal shape of a femtosecond optical pulse is altered while it propagates through a dispersive sample. By "dispersive" in this context we mean any linear system in which the propagation constant $\beta(\omega)$ as a function of frequency has any form other than a straight line through the origin, i.e., $\beta=\omega / c$. Here $\beta(\omega)$ represents the propagation characteristics of the guided mode in the channel waveguide and can be written as a Taylor expansion about its value at $\omega_{0}$ with the derivatives $\beta^{\prime} \equiv d \beta / d \omega$ and $\beta^{\prime \prime} \equiv d^{2} \beta / d \omega^{2}$ evaluated at $\omega=\omega_{0}$.

This $\beta(\omega)$, which defines the dispersion characteristics, is exactly the property we would like to measure locally. Quite naturally, we can write $v_{g}\left(\omega_{0}\right) \equiv 1 / \beta^{\prime}\left(\omega_{0}\right)$ and $v_{\phi}$ $\equiv \omega_{0} / \beta\left(\omega_{0}\right)$ for the locally measured group and phase velocity, respectively. In the following, we will show that also the GVD $\beta^{\prime \prime}$ can be retrieved from our measurement by developing an analytical model for the measurements performed with a time-resolved PSTM. As a result, interesting and useful effects such as pulse compression, pulse spreading, and pulse reshaping become accessible in the local measurement.

Dispersive broadening of optical pulses is a well-studied subject found in text books [24]. Here, we will discuss the effects of dispersion to have a starting point for modelling the measurements performed with a time-resolved PSTM. The interferometric PSTM is modeled (see Fig. 6) as a heterodyne Mach-Zehnder interferometer consisting of two single-mode fibers of unequal lengths and an air path in one arm to compensate the unequal lengths, while the photonic structure under study is present in the other path. In this, the photonic structure is a dispersive element which can have dispersive properties different from the single mode fibers. By incorporating the single-mode fibers into the model, we take into account that dispersive components other than the sample are present in both the signal and reference branches.

\section{A. Heterodyne detection}

To begin, let us consider a Mach-Zehnder interferometer with heterodyne detection. The input field $\widetilde{E}(t)$ is split by the beam splitter into the fields that travel through the two arms of the interferometer. The field through the sample is designated $\widetilde{E}_{s}(z, t)$, and the delayed field through the reference branch is called $\widetilde{E}_{r}(t-\tau)$. In this the $z$ dependence is included to represent the fact that the near-field probe is moving along the sample, and thus includes additional dispersive medium as a function of position, while $\tau$ represents the optical delay in the reference branch in the usual fashion. We use a complex representation of the fields and separate the two interfering pulses into their respective amplitudes $A$ and phases $B$,

$$
\begin{gathered}
\widetilde{E}_{s}\left(z_{s}, t\right)=A_{s}\left(z_{s}, t\right) \exp \left[i B_{s}\left(z_{s}, t\right)\right], \\
\widetilde{E}_{r}(t, \tau)=A_{r}(t, \tau) \exp (i \Delta \omega t) \exp \left[i B_{r}(t, \tau)\right],
\end{gathered}
$$

where the term $\exp (i \Delta \omega t)$ is introduced to account for the frequency shift in the reference branch due to the AO modulators. At the output of the interferometer the interference of the two fields is averaged over the response time $T_{\text {resp }}$ of the detector,

$$
\begin{aligned}
I\left(z_{s}, \tau\right) \propto & \frac{1}{T_{\text {resp }}} \int_{-T_{\text {resp }} / 2}^{T_{\text {resp }} / 2}\left[A_{r}^{2}(t-\tau)+A_{s}^{2}\left(z_{s}, t\right)+2 A_{r}(t-\tau)\right. \\
& \left.\times A_{s}\left(z_{s}, t\right) \cos \left\{B_{r}(t-\tau)-B_{s}\left(z_{s}, t\right)+\Delta \omega t\right\}\right] d t .
\end{aligned}
$$

We see that the local intensity on the detector will contain, in addition to the average intensities $\left|A_{r}\right|^{2}$ and $\left|A_{s}\right|^{2}$ associated with the two beams separately, an interference term proportional to the product of the amplitudes in the respective branches. To measure this interference term, the detected signal $I\left(z_{s}, \tau\right)$ is passed through a dual output lock-in amplifier, with a phase delay of $90^{\circ}$ between both outputs, using the AO frequency shift of $\Delta \omega$ as a reference. The phase variation due to this frequency shift is very small compared to the response time of the detector, in other words $T_{\text {resp }} \Delta \omega$ $\ll 2 \pi$, so that $\Delta \omega$ can be considered constant when evaluating the integral. This, combined with the fact that for short pulses $T_{\text {resp }} \gg \tau_{p}$ holds, yields

$$
\begin{aligned}
& V_{1}\left(z_{s}, \tau\right) \propto \int_{-\infty}^{\infty} 2 A_{r}(t, \tau) A_{s}\left(z_{s}, t\right) \cos \left[B_{r}(t, \tau)-B_{s}\left(z_{s}, t\right)\right] d t, \\
& V_{2}\left(z_{s}, \tau\right) \propto \int_{-\infty}^{\infty} 2 A_{r}(t, \tau) A_{s}\left(z_{s}, t\right) \sin \left[B_{r}(t, \tau)-B_{s}\left(z_{s}, t\right)\right] d t,
\end{aligned}
$$


for the two LIA output signals. These two signals are used to extract both the amplitude and the phase of the local optical field independently, as will be discussed in Sec. IV C. Note that although not explicitly stated above, a train of pulses arrives on the detector during the integration time of the lock-in amplifier, which as a whole generates the signal at a frequency of $\Delta \omega$. As a result, averaging takes place over many individual laser pulses that are assumed to be identical.

\section{B. Pulse propagation through a dispersive medium}

We will analyze some of the effects that can arise in pulse propagation through linear systems in terms of Gaussian pulses. Such pulses are simple, mathematically tractable, and clearly exhibit all the essential physical features [24]. We start with an optical pulse with a carrier frequency $\omega_{0}$ and a complex Gaussian envelope written in the form

$$
\widetilde{E}_{0}(t)=e^{-\widetilde{\Gamma}_{0} t^{2}+i \omega_{0} t} \Leftrightarrow \widetilde{E}_{0}(\omega)=\sqrt{\frac{\pi}{\widetilde{\Gamma}_{0}}} e^{-\left(\omega-\omega_{0}\right)^{2} / 4 \widetilde{\Gamma}_{0}},
$$

where $\widetilde{\Gamma}_{0}=a-i b$ is related to the initial pulse width $\tau_{p}$ $=[2 \ln (2) / a]^{1 / 2}$ through the parameter $a$, while the parameter $b$ is a measure for the frequency chirp on the pulse.

The effect of dispersion is best described in the frequency domain to ensure that the total spectral content of the pulse remains the same. The pulse intensities used in the experiment are low enough to prevent nonlinear processes such as self-phase modulation. Effects of frequency-dependent gain or loss in the medium will be neglected in this analysis. As a result, the spectral content of the pulses does not change in either of the branches of the interferometer. The output pulse spectrum $\widetilde{E}(\omega)$ after propagating a distance $z$ through a dispersive medium will be the input spectrum $\widetilde{E}_{0}(\omega)$ multiplied by the frequency-dependent propagation constant $\beta(\omega)$ through the system

$$
\widetilde{E}(z, \omega)=\widetilde{E}_{0}(\omega) \exp [-i \beta(\omega) z] .
$$

Note that in our case different dispersive media (sample and fiber) are present in the sample arm of the interferometer. This can simply be included in the model by multiplying the original spectrum with the different frequency-dependent propagation constants for the respective media. The result is simply a summation over the different propagation constants, which is not explicitly written down in the following analysis.

In order to allow for an analytical calculation of the propagation effects, the propagation constant $\beta(\omega)$ is written as a Taylor expansion about its value at $\omega_{0}$. With this expansion the pulse spectrum can be written as

$$
\begin{aligned}
\widetilde{E}(z, \omega)= & \sqrt{\frac{\pi}{\widetilde{\Gamma}_{0}} \exp \left[-i \beta\left(\omega_{0}\right) z-i \beta^{\prime} z\left(\omega-\omega_{0}\right)\right.} \\
& \left.-\left\{\frac{1}{4 \widetilde{\Gamma}_{0}}+\frac{i \beta^{\prime \prime} z}{2}\right\}\left(\omega-\omega_{0}\right)^{2}\right]
\end{aligned}
$$

The inverse Fourier transform of Eq. (6) describes the time evolution of the electric field of the pulse as a function of the length of the dispersive medium [24]. We carry out this transformation explicitly and obtain the output pulse after traveling an arbitrary distance $z$ through a dispersive medium

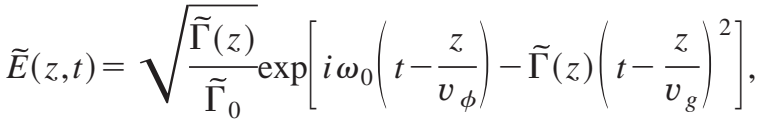

where $1 / \widetilde{\Gamma}(z)=1 / \widetilde{\Gamma}_{0}+2 i \beta^{\prime \prime} z$ is an altered pulse propagation parameter. The output pulse is still a Gaussian pulse, but with an altered pulse propagation parameter $\widetilde{\Gamma}(z)$ at the output of the system. From Eq. (7) it is clear that the carrier-frequency cycles within the pulse propagate at the phase velocity $v_{\phi}$, while the pulse envelope itself propagates at the group velocity $v_{g}$ evaluated at the center of the pulse spectrum. The pulse envelope changes in shape with distance because of the GVD $\beta^{\prime \prime}$, which is basically a variation of group velocity with frequency.

\section{Analytical model of a time-resolved PSTM}

In this section we derive an analytical model for the use of coherent detection methods to locally measure the time evolution of a short light pulse. In our experiment unchirped Fourier limited pulses are coming from the laser, so that $\widetilde{\Gamma}_{0}$ $\in \mathbb{R}$. To write the signal measured by the lock-in amplifier [Eq. (3)] in terms of short laser pulses, we start the analysis by separating Eq. (7) for a pulse propagating through a dispersive medium in its respective amplitude and phase,

$$
\begin{aligned}
\widetilde{E}(z, t)= & \frac{1}{\sqrt[4]{1+\left(2 \beta^{\prime \prime} z \Gamma_{0}\right)^{2}}} \exp \left[-\frac{\Gamma_{0}}{1+\left(2 \beta^{\prime \prime} z \Gamma_{0}\right)^{2}}\right. \\
& \left.\times\left(t-\frac{z}{v_{g}}\right)^{2}\right] \exp \left[\frac{i}{2} \arctan \left(-2 \beta^{\prime \prime} z \Gamma_{0}\right)\right. \\
& \left.+i \omega_{0}\left(t-\frac{z}{v_{\phi}}\right)+\frac{2 i \beta^{\prime \prime} z \Gamma_{0}^{2}}{1+\left(2 \beta^{\prime \prime} z \Gamma_{0}\right)^{2}}\left(t-\frac{z}{v_{g}}\right)^{2}\right] .
\end{aligned}
$$

From this equation it can clearly be seen that as a result of the GVD, the pulse is broadened upon propagation through the dispersive medium. In fact, the factor [1 $\left.+\left(2 \beta^{\prime \prime} z \Gamma_{o}\right)^{2}\right]^{1 / 2}$ corresponds to the broadening of the FWHM of pulses upon propagation through a dispersive medium. The phase, i.e., the imaginary part in Eq. (8), contains a quadratic time dependence which corresponds to a linear frequency chirp in the pulse due to the GVD. Note that in contrast to Eq. (7) the above equation is only valid for $\widetilde{\Gamma}_{0}$ $\in \mathbb{R}$ as this simplifies the mathematics considerably; although Eq. (8) could as well be derived for a complex $\Gamma_{0}$, this is not needed for our unchirped input pulses. 
By substituting the expression for a pulse propagating through a dispersive medium in Eq. (3) for the respective branches of the interferometer, we obtain

$$
\begin{aligned}
V_{1}\left(z_{s}, \tau\right) \propto A & \int_{-\infty}^{\infty} \exp \left[-\Gamma_{r}\left(t-\xi_{r}\right)^{2}-\Gamma_{s}\left(t-\xi_{s}\right)^{2}\right] \\
& \times \cos \left\{\Upsilon+\kappa_{r} \Gamma_{r}\left(t-\xi_{r}\right)^{2}+\kappa_{s} \Gamma_{s}\left(t-\xi_{s}\right)^{2}\right\} d t,
\end{aligned}
$$

where

$$
\begin{gathered}
\kappa_{r}=2 \beta_{r}^{\prime \prime} z_{r} \Gamma_{0}, \quad \kappa_{s}=-2 \beta_{s}^{\prime \prime} z_{s} \Gamma_{0}, \\
\Gamma_{r}=\frac{\Gamma_{0}}{1+\left(\kappa_{r}\right)^{2}}, \quad \Gamma_{s}=\frac{\Gamma_{0}}{1+\left(\kappa_{s}\right)^{2}}, \\
A=\frac{A_{r} A_{s 4}}{\sqrt{\Gamma_{0}}} \sqrt{\Gamma_{r} \Gamma_{s}}, \\
\xi_{r}=\tau+\frac{z_{r}}{v_{g r}}, \quad \xi_{s}=\frac{z_{s}}{v_{g s}},
\end{gathered}
$$

and

$$
\Upsilon=-\frac{\arctan \left(\kappa_{r}\right)+\arctan \left(\kappa_{s}\right)}{2}-\omega_{0}\left(\tau+\frac{z_{r}}{v_{\phi r}}-\frac{z_{s}}{v_{\phi s}}\right) .
$$

Here, $A$ corresponds to an amplitude with $A_{r}$ and $A_{s}$ constants that correct for the different optical power density in the two branches, $\Gamma_{r}, \Gamma_{s}$ to the real part of a modified pulse propagation parameter, $\xi_{r}, \xi_{s}$ to an optical path length, $\kappa_{r}, \kappa_{s}$ to a GVD dependent parameter, and $Y$ is simply a timeindependent variable in the optical phase. To interpret this mathematical result, we carry out the integration explicitly using the following standard integral [25]:

$$
\begin{aligned}
\int_{-\infty}^{\infty} \exp \left[-\left(a x^{2}+2 b x+c\right)\right] \cos \left(p x^{2}+2 q x+r\right) d x \\
=\frac{\sqrt{\pi}}{\sqrt[4]{a^{2}+p^{2}}} \exp \left[\frac{a\left(b^{2}-a c\right)-\left(a q^{2}-2 b p q+c p^{2}\right)}{a^{2}+p^{2}}\right] \\
\quad \times \cos \left\{\frac{\arctan (p / a)}{2}\right. \\
\left.-\frac{p\left(q^{2}-p r\right)-\left(b^{2} p-2 a b q+a^{2} r\right)}{a^{2}+p^{2}}\right\} \quad[a>0] .
\end{aligned}
$$

For the other channel of the LIA, the cosine in Eqs. (9a) and (10) can simply be replaced by the sine term [25]. For our purposes it is convenient to write this equation directly in terms of its respective magnitude $\mathcal{A}$ and phase $\Phi$ in the following manner:

$$
\begin{aligned}
\mathcal{A} & =\sqrt{V_{1}\left(z_{s}, \tau\right)^{2}+V_{2}\left(z_{s}, \tau\right)^{2}} \\
& =\frac{\sqrt{\pi}}{\sqrt[4]{a^{2}+p^{2}}} \exp \left[\frac{a\left(b^{2}-a c\right)-\left(a q^{2}-2 b p q+c p^{2}\right)}{a^{2}+p^{2}}\right],
\end{aligned}
$$

and

$$
\begin{aligned}
\Phi & =\arccos \left[\frac{V_{1}\left(z_{s}, \tau\right)}{\mathcal{A}}\right] \\
& =\left\{\frac{\arctan (p / a)}{2}-\frac{p\left(q^{2}-p r\right)-\left(b^{2} p-2 a b q+a^{2} r\right)}{a^{2}+p^{2}}\right\},
\end{aligned}
$$

where the $V_{1}\left(z_{s}, \tau\right), V_{2}\left(z_{s}, \tau\right)$ are the two LIA output channels and show the relation with the measured signals. Substituting

$$
\begin{gathered}
a=\Gamma_{r}+\Gamma_{s}, \quad b=-\Gamma_{r} \xi_{r}-\Gamma_{s} \xi_{s}, \\
c=\Gamma_{r} \xi_{r}^{2}+\Gamma_{s} \xi_{s}^{2}, \quad p=\Gamma_{r} \kappa_{r}+\Gamma_{s} \kappa_{s}, \\
q=-\Gamma_{r} \xi_{r} \kappa_{r}-\Gamma_{s} \xi_{s} \kappa_{s}, \quad r=\Gamma_{r} \xi_{r}^{2} \kappa_{r}+\Gamma_{s} \xi_{s}^{2} \kappa_{s}+\Upsilon
\end{gathered}
$$

in Eqs. (11) and (12) and some minor rewriting yields the expressions for the amplitude and phase of the pulse as measured by the time-resolved PSTM,

$$
\mathcal{A}\left(z_{s}, \tau\right)=\frac{A_{r} A_{s} \sqrt{\pi / \Gamma_{0}}}{\sqrt[4]{4+\left(\kappa_{r}+\kappa_{s}\right)^{2}}} \exp \left[\frac{-2 \Gamma_{0}\left(\xi_{r}-\xi_{s}\right)^{2}}{4+\left(\kappa_{r}+\kappa_{s}\right)^{2}}\right]
$$

and

$$
\begin{aligned}
\Phi\left(z_{s}, \tau\right)= & \Upsilon+\frac{1}{2} \arctan \left\{\frac{\Gamma_{r} \kappa_{r}+\Gamma_{s} \kappa_{s}}{\Gamma_{r}+\Gamma_{s}}\right\} \\
& -\left\{\frac{\Gamma_{0}\left(\xi_{r}-\xi_{s}\right)^{2}\left[\kappa_{r}+\kappa_{s}\right]}{4+\left(\kappa_{r}+\kappa_{s}\right)^{2}}\right\},
\end{aligned}
$$

where the variables with the subscript $s$ are the ones that change as a function of the position of the probe. Note that the optical delay line is kept at a fixed position for each individual measurement, so that $\tau$ is, in fact, constant.

With Eq. (14), we have an analytical expression for the measured pulse amplitude. It can be seen that the measured length of the pulse (see Fig. 2) in the waveguide is influenced by both the original pulse length as well as by the GVD in the two branches of the interferometer. To get a more qualitative feeling for the influence of the GVD on the measured FWHM, we depicted the optical amplitudes calculated by Eq. (14) for different values of the GVD ranging from 0 to $150 \mathrm{ps}^{2} / \mathrm{km}$ in Fig. 7. In this calculation the measured fiber lengths in both branches, as given earlier, were used as an input. In Fig. 7 we see Gaussian pulse envelopes, for which the FWHM increases as function of the amount of GVD in the system. From the resulting Gaussian shape we can conclude that the pulse shape is almost completely de- 


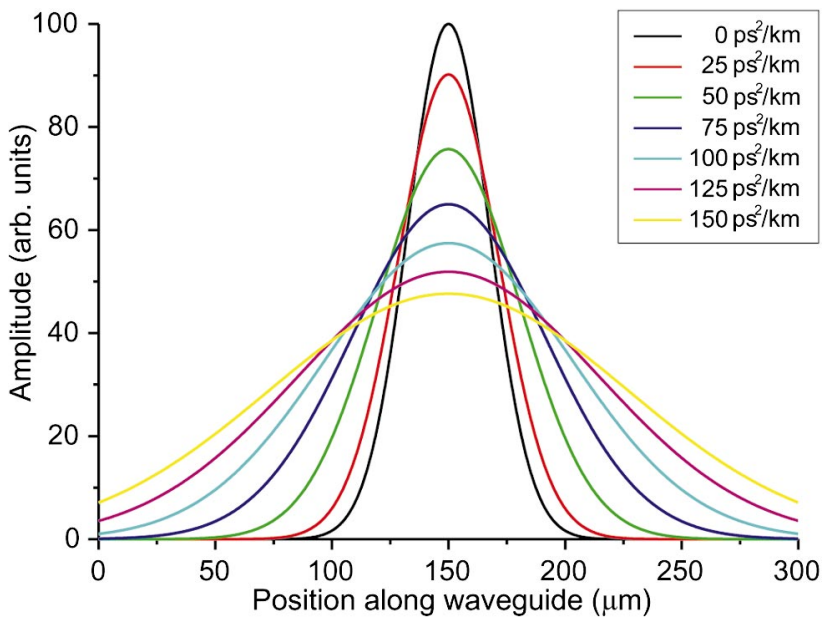

FIG. 7. (Color) Calculated optical amplitude [Eq. (14)] for our experimental setup for different values of the GVD in our system. The GVD parameter ranges from 0 to $150 \mathrm{ps}^{2} / \mathrm{km}$ in steps of $25 \mathrm{ps}^{2} / \mathrm{km}$. Other experimental parameters used in this calculation are given in the text. A Gaussian pulse shape can be observed, for which the FWHM strongly depends on the group velocity dispersion in the two branches of the interferometer.

termined by the exponent in Eq. (14). Therefore, we can write the following for the FWHM of the Gaussian pulse:

$$
\tau_{\text {pulse }}\left(z_{s}\right)=v_{g s} \sqrt{\frac{\ln 2\left[4+\left(\kappa_{r}+\kappa_{s}\right)^{2}\right]}{\Gamma_{0}}},
$$

measured along the propagation direction. From this expression it is clear that the measured FWHM is given by the original pulse length corrected for the GVD in the two branches of the interferometer. Note that the $\kappa_{r}$ and $\kappa_{s}$ as given by Eq. (9b) are opposite in sign. So if the optical fibers in the two branches are equal in length and have the same GVD, their effect on the measured FWHM of the amplitude and phase cancels, as is obvious from Eqs. (14) and (15). The term in front of the exponential in Eq. (14) is found to induce a slight asymmetry in the Gaussian shape, only visible when very large values for the GVD are used as input for the calculations. In the experimental setup, an additional inclusion of $300 \mu \mathrm{m}$ equivalent to the total scanrange in the sample branch yields a negligible reduction of the prefactor of only $0.0395 \%$ for a GVD of $75 \mathrm{ps}^{2} / \mathrm{km}$.

However, as can be seen in Fig. 7, the maximum visible amplitude of the pulses reduces as a function of the GVD due to this prefactor. To get a feeling for this dependence, Fig. 8 shows the calculated visibility of the fringe pattern for the fiber length difference between the two branches using different values of the GVD ranging from 0 to $300 \mathrm{ps}^{2} / \mathrm{km}$. The amplitude at the center of the pulse when no dispersive medium is present in either of the two branches is defined as $100 \%$ visibility. Plotted is the amplitude at the center of the pulse for different amounts of dispersive medium. It is clear that the stretching of the pulses in the two branches has a strong influence on the visibility of the fringes. Note that an equal amount of dispersive medium in both branches has no

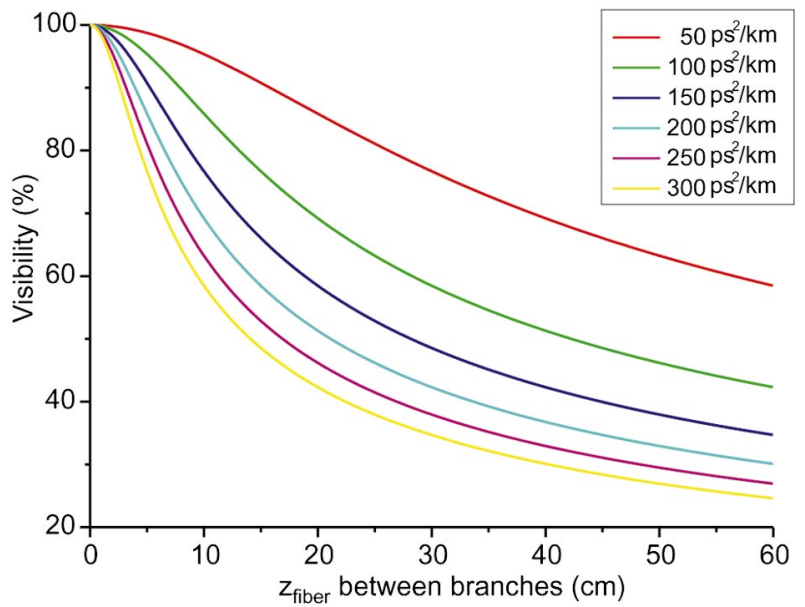

FIG. 8. (Color) The imbalance between the amount of dispersive media in the two branches of the interferometer has a strong influence on the visibility of the fringe pattern as can be seen in this calculation. Depicted is the calculated visibility of the fringe pattern as a function of $\Delta z_{\text {fiber }}$ (see Fig. 6), using values of the GVD ranging from 0 to $300 \mathrm{ps}^{2} / \mathrm{km}$. For optimal detection efficiency, the dispersive media in the two branches should clearly be balanced.

influence on the detected signal. For optimal detection efficiency, the dispersive media in the two branches should clearly be balanced.

As we are interested in the local optical properties of the photonic structure, we started this discussion with the fact that the photonic structure is present in the sample arm of the interferometer. The different material contributions due to the $\beta(\omega)$ can simply be added as can be seen from Eq. (5). So if the two fibers and other dispersive elements (excluding the sample itself) are equal in the two branches, then the only

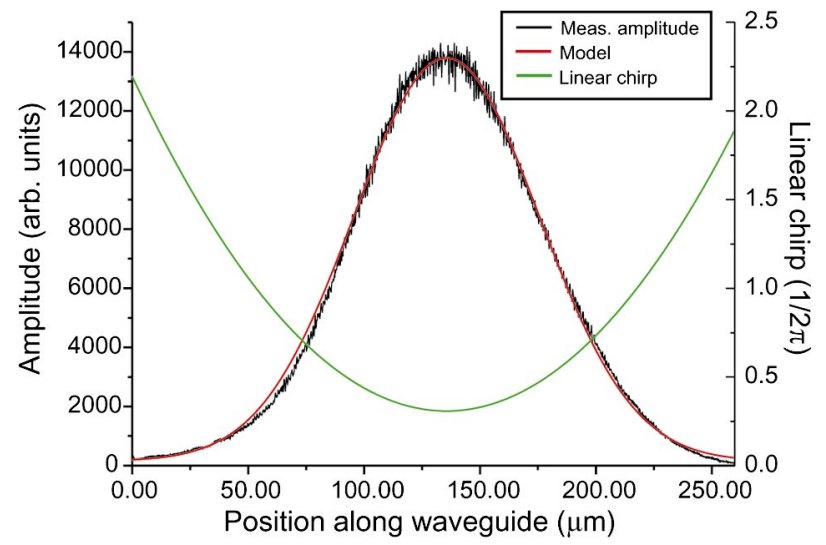

FIG. 9. (Color) Direct comparison between the measured (solid black line) and calculated (red line) optical amplitude. An excellent agreement is obtained for a GVD parameter of $72.4 \mathrm{ps}^{2} / \mathrm{km}$ as can be seen here. The calculated phase [Eq. (15)] neglecting the rapid oscillating term around $\omega_{0}$ for this GVD parameter shows that a small chirp in the fringes is to be expected in the measurement. The right axis represents the amount of periods changed due to the linear chirp. This expected chirp is small compared to the current nonlinearity in the probe movement. 
remaining contribution due to the GVD is from the sample itself. Therefore, the GVD of the structure under study can directly and locally be determined from the locally measured amplitude and optical phase of the pulse. Even if branches are not compensated (excluding the sample), a reference measurement without the sample would yield the dispersion characteristics of the setup such that a direct fit using Eq. (14) would yield the local group velocity dispersion of the sample.

\section{DISCUSSION}

In the experiment the $123 \pm 3$ fs pulse travels through dispersive fibers and other glass components in the two arms of the interferometer. In Eq. (16) we have seen that the FWHM of the pulse along the waveguide is determined by the differences between the two branches and the original pulse length. In the experiment we have measured a FWMH along the propagation direction of $66.8 \mu \mathrm{m}$ [see Fig. 2(c)]. Under the assumption of a homogeneous GVD in our system, given the difference in fiber length of $\approx 30 \mathrm{~cm}$, we can reproduce the measured results with a GVD value of $72.4 \mathrm{ps}^{2} / \mathrm{km}$. A direct comparison for the measured and calculated pulse amplitude is given in Fig. 9. The black line in this graph shows the measured optical amplitude through the heart of the waveguide given in Fig. 2(c), while the red line represents the calculated amplitude. It is clear that an excellent agreement is obtained between experiment and theory. The measured GVD value is in the range expected for single-mode fibers for infrared wavelengths, although the value appears to be high, as typical values are smaller then $20 \mathrm{ps}^{2} / \mathrm{km}$ [26]. This high value could be explained by the fact that other optical components with unknown dispersion characteristics are also presents in both the branches.

As a result of the GVD, the pulses in the reference and sample branches have been stretched considerably. The calculated value for the GVD shows that the nearly Fourier limited $123 \pm 3$ fs laser pulses have lengthened to a $1144 \mathrm{fs}$ signal pulse and a 640 fs reference pulse by the time they interfere with each other in the fiber coupler of the interferometric PSTM. The stretching of the pulses has a large influence on the visibility of the signal as demonstrated by Fig. 8 . In the experiment the pulses propagate through 69.7 $\pm 0.5 \mathrm{~cm}$ and $38.5 \pm 0.5 \mathrm{~cm}$ of dispersive fibers and other glass components in the two branches, respectively. Based on the theoretical model, the visibility in the experiment was therefore reduced to about $65 \%$ at the time of measurements. Balancing the dispersive media in the two branches will clearly lead to an increase of the visibility.

Until now, we have mostly discussed the measured amplitude of the pulses. However, our detection scheme is fully interferometric, so information about both amplitude and optical phase is obtained. As demonstrated, the distance between the fringes is a direct measure for the local wavelength and therefore yields the phase velocity. The expression [Eq. (15)] for the optical phase of the pulse, however, contains a quadratic term corresponding to a linear frequency chirp in the pulse. In Fig. 9 we also plotted the calculated phase [Eq. (15)] divided by $2 \pi$ while neglecting the rapid oscillating term around $\omega_{0}$ from $\Upsilon$ [see Eq. (9b)]. The total phase change within the pulse duration for the experimental configuration is roughly 0.1 periods over the measured FWHM of $66.8 \mu \mathrm{m}$ which corresponds to 77 periods of the optical field. In other words, the periodicity in the center of the pulse with respect to the wings would differ by only $2-4 \mathrm{~nm}$. To directly measure the linear chirp on the optical pulse, a small difference of $0.1-0.3 \%$ in the periodicity must be resolved. In principle, such small differences can be directly measured, however, a highly linear scan system is required. At this stage the linearity of the probe movement in our setup, which has a position feedback system to compensate for the nonlinearity of the piezoelectric scanner, is of the same order of magnitude (less than $0.2 \%$ ).

It is tempting to consider the information obtained by the time-resolved measurements performed in this paper as a conventional cross correlation of the optical fields in the reference branch and the photonic structure, similar to earlier work using phase-sensitive time-resolved interferometry $[1,15]$. However, this notion is too simplistic. The pulse envelope in the waveguide propagates at a speed different from the phase information, and therefore the position on the photonic structure cannot be translated to a single time delay $\tau$ for the cross-correlation function. This is in contrast to pulse propagation in air, for which both the group and phase velocities are the same. The difference between moving the probe or the optical delay can also be seen in the theoretical model in Eqs. (14) and (15). A movement of the probe movement leads to an increase of $\kappa_{s}$. However, by moving only the optical delay line, $\kappa_{r}$ will remain constant as no additional dispersion is included as a function of the movement. This clearly demonstrates the effect of including additional dispersive medium on the measured optical signal as a function of the probe movement with respect to the measurements performed by moving the optical delay line in air, i.e., changing $\tau$ as in conventional phase-sensitive time-resolved interferometry.

\section{CONCLUSION}

In conclusion, the propagation in time of a nearly Fourier limited laser pulse propagating through a channel waveguide has been visualized by an interferometric PSTM. The local amplitude and phase of the pulse have been retrieved so that both the phase and group velocity could be measured locally. The observed length of the measured pulse envelope is explained by comparison with an analytical model derived in this paper. The observed FWHM of the measured pulse shape can be attributed to the group velocity dispersion in the fibers that are unequal in length for the two branches of the interferometer.

The model shows that by balancing the amount of dispersive medium in the two branches of the interferometer it becomes possible to locally measure the GVD of the structure under study. Even if branches are not compensated, a reference measurement makes it possible to measure group 
velocity dispersion locally in a sample. As a result, interesting and useful effects such as pulse compression, pulse spreading, and pulse reshaping become accessible in the local measurement.

It is expected that the time-resolved interferometric PSTM will, in the near future, be used for the local experimental investigation of predicted physical phenomena inside (non)linear dispersive media, such as integrated waveguide structures and photonic crystals.

\section{ACKNOWLEDGMENTS}

This research was part of the strategic Research Orientation on Advanced Photonic Structures of the $\mathrm{MESA}^{+} \mathrm{Re}-$ search Institute. Furthermore, this work was part of the research program of the Stichting voor Fundamenteel Onderzoek der Materie [FOM, financially supported by the Nederlandse Organisatie voor Wetenschappelijk Onderzoek (NWO)].
[1] J.C. Diels and R.W. Wolfgang, Ultrashort Laser Pulse Phenomena (Academic, San Diego, 1996).

[2] Y. Sugimoto et al., Appl. Phys. Lett. 81, 1946 (2002).

[3] A. Yariv, Y. Xu, R. Lee, and A. Scherer, Opt. Lett. 24, 711 (1999).

[4] M. Notomi et al., Phys. Rev. Lett. 87, 253902 (2001).

[5] K. Inoue et al., Phys. Rev. B 65, 121308 (2002).

[6] Photonic Crystals and Light Localization in the 21st Century, Vol. 563 of NATO Advanced Studies Institute, Series $C$, edited by C.M. Soukoulis (Kluwer Academic, Dordrecht, The Netherlands, 2001).

[7] B.J. Eggleton et al., Phys. Rev. Lett. 76, 1627 (1996).

[8] H.G. Winful and V. Perlin, Phys. Rev. Lett. 84, 3586 (2000).

[9] M. Mlejnek, E.M. Wright, J.V. Moloney, and N. Bloembergen, Phys. Rev. Lett. 83, 2934 (1999).

[10] W. Nakagawa et al., J. Opt. Soc. Am. A 18, 1072 (2001).

[11] M. Scalora, J.P. Dowling, A.S. Manka, C.M. Bowden, and J.W. Haus, Phys. Rev. A 52, 726 (1995).

[12] W. Chen and D.L. Mills, Phys. Rev. Lett. 58, 160 (1987).

[13] Ch. Spielmann, R. Szipöcs, A. Stingl, and F. Krausz, Phys. Rev. Lett. 73, 2308 (1994).

[14] J.K. Ranka, R.W. Schirmer, and A.L. Gaeta, Phys. Rev. Lett. 77, 3783 (1996).
[15] A. Imhof, W.L. Vos, R. Sprik, and A. Lagendijk, Phys. Rev. Lett. 83, 2942 (1999).

[16] M.D. Rahn, A.M. Fox, M.S. Skolnick, and T.F. Krauss, J. Opt. Soc. Am. B 19, 716 (2002).

[17] M.C. Netti et al., Appl. Phys. Lett. 81, 3927 (2002).

[18] For a classical example, see K. Smith and L.F. Mollenauer, Opt. Lett. 14, 1284 (1989).

[19] M.L.M. Balistreri et al., Science 294, 1080 (2001).

[20] M.L.M. Balistreri, J.P. Korterik, L. Kuipers, and N.F. van Hulst, Phys. Rev. Lett. 85, 294 (2000).

[21] E. Flück et al., J. Microsc. 202, 104 (2001).

[22] A. Nesci, R. Dändliker, and H.P. Herzig, Opt. Lett. 26, 208 (2001).

[23] M.L.M. Balistreri, J.P. Korterik, L. Kuipers, and N.F. van Hulst, J. Lightwave Technol. LT19, 1169 (2001).

[24] A.E. Siegman, Lasers (University Science Books, Sausalito, 1986).

[25] Table of Integrals, Series, and Products, edited by I.S. Gradshteyn, I.M. Ryzhik, and Alan Jeffery (Academic, San Diego, 1994).

[26] G.P. Agrawal, Nonlinear Fiber Optics, 3rd ed. (Academic, San Diego, 2001). 